\title{
MAGNETIC FIELDS, OSCILLATIONS, AND HEATING IN THE \\ QUIET SUN TEMPERATURE MINIMUM REGION FROM

\author{
ULTRAVIOLET OBSERVATIONS AT $1600 \AA$
}

\author{
J. W. COOK \\ E.O. Hulburt Center for Space Research, Naval Research Laboratory, \\ Washington, DC 20375, U.S.A.
}

\begin{abstract}
The High Resolution Telescope and Spectrograph (HRTS) instrument has obtained broadband spectroheliograph images at $1600 \AA$ of the solar temperature minimum region. I discuss HRTS observations of quiet areas and their relation with magnetic fields, five minute oscillations, and heating. The brightness temperature of solar fine structure elements composing the supergranular net work is found to be linearly proportional to the local absolute value of magnetic field strength. There is evidence for a 250-s period oscillation occurring in 10-arcsec scale patches, which however is energetically unimportant to the local heating budget. A general nonmagnetic background heating and five minute oscillations occur globally, while the net work bright points occur in magnetic regions, heated perhaps from partial dissipation of Alfvén waves (whose energy flux is linearly proportional to $B$ ) in individual elemental 1500-G (at the photosphere) flux tubes which expand to form the temperature minimum fine structure bright points.
\end{abstract}

Key words: flux tubes - infrared:stars - Sun: magnetic fields - Sun: UV radiation

\section{Introduction}

The temperature minimum region of the solar atmosphere can be observed at infrared wavelengths both in the continuum around $350 \mu \mathrm{m}$, and in absorption lines such as the vibration-rotation lines of $\mathrm{CO}$, for example the 3-2 R(14) line at $4.67 \mu \mathrm{m}$. This region can also be observed in the far ultraviolet, and observations as far back as 1978 from sounding rocket flights have been made with arcsecond spatial resolution. A comparison with such observations can lead to a fuller understanding of the temperature minimum.

The High Resolution Telescope and Spectrograph (HRTS) instrument consists of a 30-cm diameter telescope, a broadband spectroheliograph, a slit spectrograph which can cover a wavelength range from $1175-1710 \AA$ with $0.05 \AA$ spectral resolution, and an $\mathrm{H} \alpha$ system which can both display images using a TV camera and record them on film. Both the spectroheliograph and $\mathrm{H} \alpha$ systems use reflected images from the spectrograph slit jaw mirrors. Slit spectra and spectroheliograph images are recorded on film. The spatial resolution, while potentially an arcsecond or better, has typically been 1-2 arcsec.

The spectroheliograph has been tuned on a number of sounding rocket flights to cover a passband centered on $1600 \AA$, where the predominant flux contributor is continuum emission from the temperature minimum region (approximately $70 \%$ of the integrated intensity over the passband in disk quiet regions; the remainder is chromospheric line emission). I discuss HRTS observations from quiet areas of bright point fine structure, its relation to magnetic fields seen in photospheric magnetograms, the evidence for five minute oscillations, and estimates of required heating. Where available, I compare results from the far ultraviolet observations with work in the infrared, noting both similarities and also several puzzling differences. 


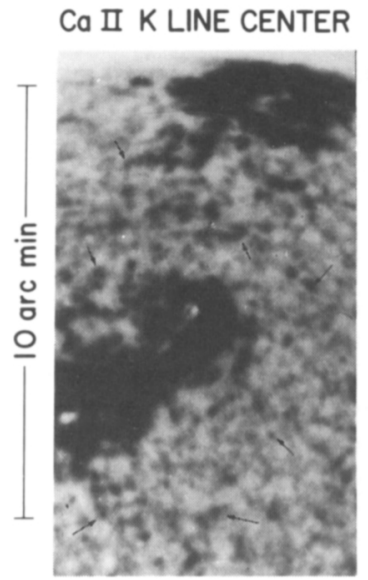

(a)

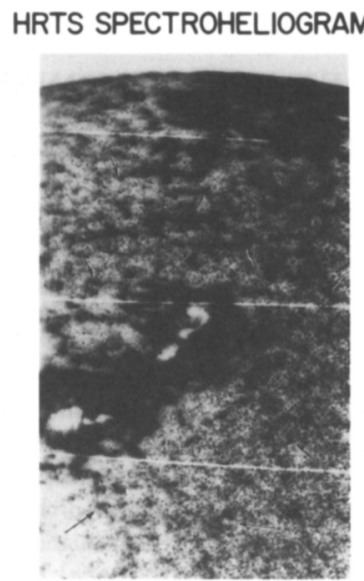

(b)

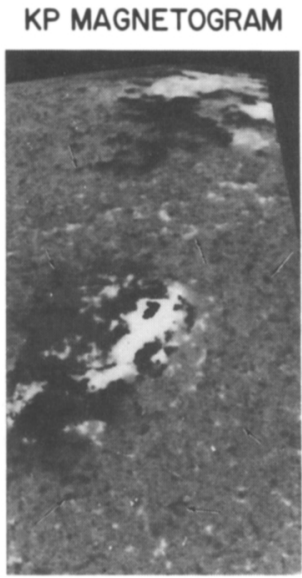

(c)

Fig. 1. Comparison of Sac Peak Ca II image, HRTS $1600 \AA$ spectroheliogram, and Kitt Peak magnetogram from the day of the HRTS II flight.

\section{Structure of Quiet Regions}

The central problem of the physics of the solar atmosphere is to understand the physical processes which reverse the temperature decline in the upper photosphere, as consistent with radiative equilibrium, and instead lead to the temperature rise from the temperature minimum at $4400 \mathrm{~K}$, through the chromosphere and transition region, up to the several times $10^{6} \mathrm{~K}$ corona. This input of additional energy is clearly connected with the magnetic field, either as a channeling agency, such as for magneto-acoustic waves, or more directly through input of energy from changes in the magnetic field such as reconnection.

The observed structure of the solar photosphere is a granular pattern with individual granules of order 1-2 arcsec in size which rise and fall vertically, have sideways motions of order $1 \mathrm{~km} \mathrm{~s}^{-1}$, and last around 8 minutes. But by the temperature minimum, where the atmosphere already departs from a radiative equilibrium model and additional heating is occuring (Anderson and Athay 1990), the observed structure in quiet areas is the supergranular network.

Small network elements composed of clumped arcsec scale bright points occur at edges of the supergranulation, coincident with stronger field regions in photospheric magnetograms, while the cell centers are filled with the order of 20 individual bright points evolving on a 1 minute timescale (Cook, Brueckner, and Bartoe 1983). Figure 1 illustrates a Ca II image, HRTS $1600 \AA$ spectroheliogram, and photospheric magnetogram from the day of the HRTS II flight on 13 February 1978, showing the similarity of structure in Ca II and the $1600 \AA$ image, and the relationship of bright areas (dark in these negative images) with strong field regions in the magnetogram (Cook, Brueckner, and Bartoe 1983). In comparison, a new, unpublished image at $350 \mu \mathrm{m}$ obtained by the JCMT was shown at this meeting, with a spatial resolution of approximately $10 \mathrm{arcsec}$, which only begins to suggest the network element 
supergranular structure of quiet areas (Lindsey 1993).

This supergranular network structure of quiet regions can be observed throughout the chromosphere and into the transition region, with a good degree of correspondence in location of network elements in images from a range of temperatures. This basic network structure persists up to at least $500,000 \mathrm{~K}$, as can be seen in Skylab data from the NRL SO82A instrument (Sheeley et al. 1975; Cook 1991). Images in $\mathrm{Ne}$ VII, formed at $500,000 \mathrm{~K}$, show that this temperature represents something of a transitional range for solar morphology. The quiet disk is still organized in the network pattern, but loop structures are beginning to appear in active regions. At temperatures of $10^{6} \mathrm{~K}$ (seen in Mg IX) and higher (Fe XV), the disk and limb show large complete loop systems, typically connecting separated active regions, and the supergranular pattern has disappeared. The basic quiet Sun structure up to at least $500,000 \mathrm{~K}$ is closely related to structures of lower temperature plasmas, instead of to the corona.

\section{Relationship of Brightness and Magnetic Flux}

Using HRTS $1600 \AA$ spectroheliograms and Kitt Peak magnetograms, Cook and Ewing (1990) examined the quantitative relationship of brightness temperature at $1600 \AA$ to photospheric magnetic field strength in the quiet Sun. We used a technique which obtained the best-fit relationship of a given functional form between two histogram distributions, in our case for brightness temperature at $1600 \AA$ and for the distribution of absolute magnetic flux, in a $486 \times 452 \operatorname{arcsec}^{2}$ sample quiet area from the HRTS V flight on 11 December 1987.

Figure 2 illustrates the distribution of brightness temperature at $1600 \AA$ which we found for our sample quiet area (the solid and dashed lines were obtained from two different exposures of the same sample area). The functional form used for mapping one distribution to the other is very general, and was originally meant to reproduce the $\mathrm{S}$-curve shape of film characteristic curves in photometric photometry (see Cook, Ewing, and Sutton 1988). The observations alone determined the fit, which was not imposed at the start. In Figure 3 we show the functional relationship which we found (the solid and dashed lines from two different $1600 \AA$ exposures of the same sample area are again in good agreement), which is essentially linear for field strengths above the $\sim 7 \mathrm{G}$ noise level of the Kitt Peak magnetograms. We found that even magnetic bipoles, unlike in the corona, are no brighter than implied by their absolute flux magnitude.

We estimated the additional energy flux necessary to maintain the network bright points by integrating the LTE approximation $\Delta E=16 \sigma T_{0}^{3} \Delta T \tau$, where $\Delta T$ is the observed brightness temperature excess in network bright points above an average temperature $\mathrm{T}_{0}$, and $\tau$ is the optical depth (at $5000 \AA$ ) at the temperature minimum level, over the observed distribution of brightness temperature above $4600 \mathrm{~K}$, which represents the bright point population. This average additional energy flux, $7.5 \times 10^{6} \mathrm{ergs} \mathrm{cm}^{-2} \mathrm{~s}^{-1}$, is directly comparable to the flux required to heat the normal average global background at the $T_{\min }$ level, $8 \times 10^{6} \mathrm{ergs} \mathrm{cm}^{-2}$ $\mathrm{s}^{-1}$, from the prescribed heating models of Anderson and Athay (1990).

This linear result is consistent with heating by Alfvén waves, whose flux $F_{A}=$ 


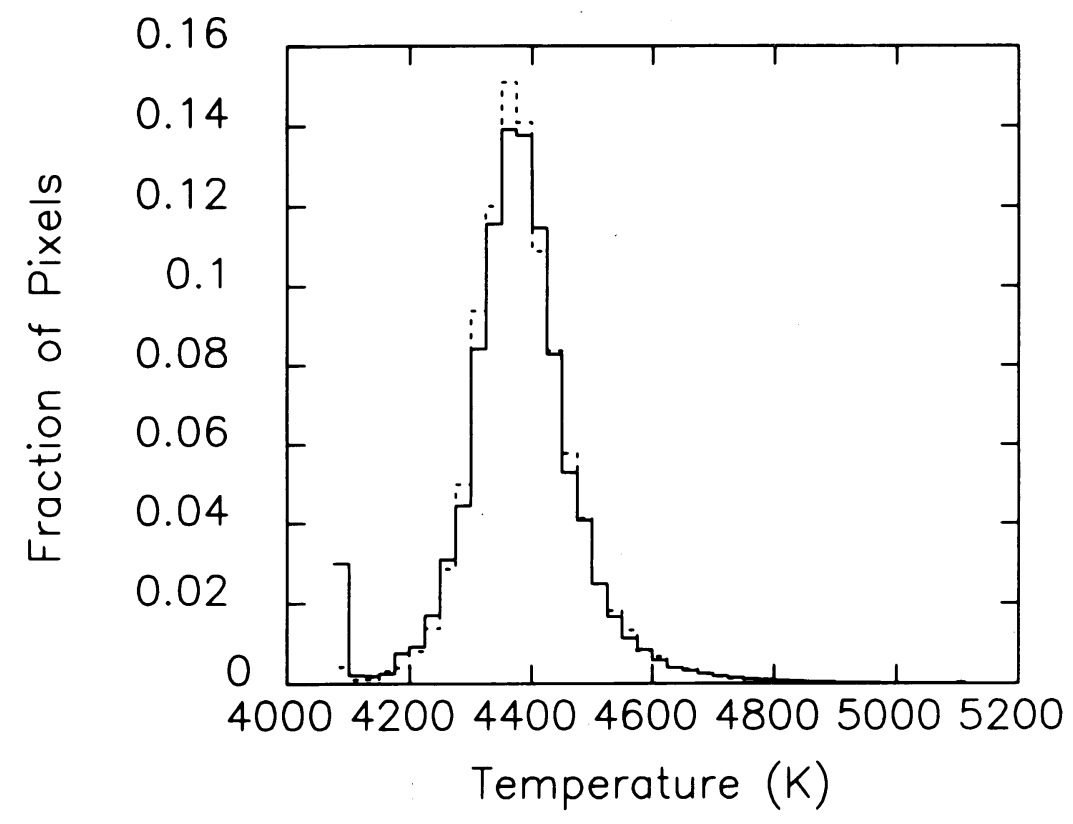

Fig. 2. Histogram distribution of brightness temperature in a sample quiet area.

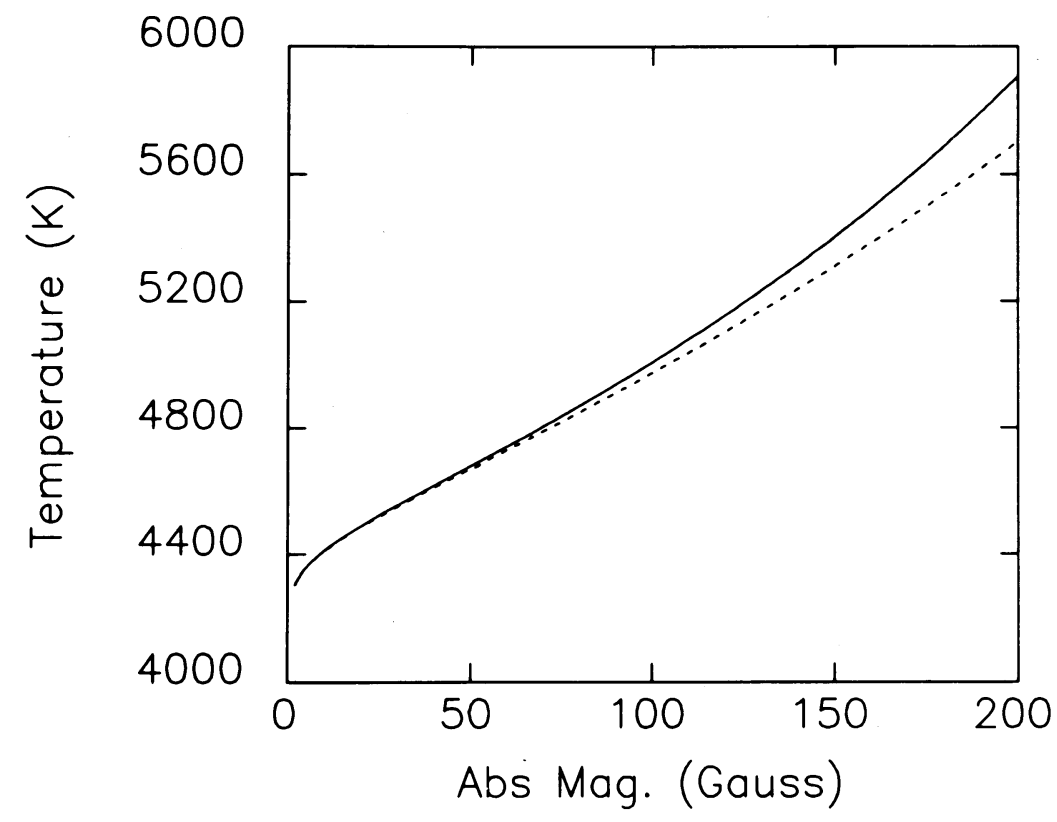

Fig. 3. Relationship of $1600 \AA$ brightness temperature vs. absolute magnetic field strength in a sample quiet area. 
$\rho v^{2} V_{A}$, where $V_{A}=B /(4 \pi \rho)^{1 / 2}$ is the Alfvén velocity, is linear in $B$. We estimated the potential flux at photospheric levels produced from granular buffeting in magnetized areas. Using $v \sim 1 \mathrm{~km} \mathrm{~s}^{-1}$ as the typical horizontal granular velocity (Title et al. 1989) and $\rho=2.7 \times 10^{-7} \mathrm{~g} \mathrm{~cm}^{-3}$ as the density at the $\tau=1$ level of the photosphere (Vernazza, Avrett, and Loeser 1981), there is 10 times the flux required for heating the $T_{\min }$ network element bright points potentially available in Alfvén waves generated from photospheric granular buffeting. The problem still remains, however, to find a viable dissipation mechanism for the $T_{\min }$ region.

Our comparison with the energy available in Alfvén waves was suggested by the observed linear relationship of excess heating to magnetic flux, but we did not mean to rule out other mechanisms which could yield such a result.

Cook and Ewing (1990) suggested that the individual network bright points of $\sim 1$ arcsec diameter seen at $1600 \AA$, associated with typical Kitt Peak observed field strengths of $50 \mathrm{G}$ obtained for 2 arcsec seeing, were consistent with individual flux tubes with true, unresolved photospheric field strengths of around $1800 \mathrm{G}$ and diameters of $\sim 1 / 3$ arcsec. They used a simple scaling law for the diameter $d$ of a flux tube based on conservation of flux and equipartition of magnetic pressure and gas pressure $p,\left(d_{2} / d_{1}\right)=\left(p_{1} / p_{2}\right)^{1 / 4}$. The VAL model $\mathrm{C}$ atmosphere (Vernazza, Avrett, and Loeser 1981) gives the ratio of gas pressures at $\tau=1$ and the $T_{\min }$ as 88 , and so $d\left(T_{\min }\right) \sim 3.1 d$ (photosphere). A field of $50 \mathrm{G}$ at $2 \operatorname{arcsec}$ resolution then corresponds to $50 \times 36=1800 \mathrm{G}$ at $1 / 3$ arcsec. Magnetograms from the Lockheed SOUP instrument (Title et al. 1990) have reached 0.5 arcsec spatial resolution, and found that field strengths associated with $\mathrm{Ni}$ I bright points are of the order of $600 \mathrm{G}$. Spatial resolution below 0.5 arcsec should show even higher field strengths for elementary photospheric flux tubes. The $T_{\min }$ is the highest level where network and active region features still keep a resolvable bright point structure. At greater heights the decreasing gas pressure and expanding fields cause the pointlike network features to spread out to more continuous patches of emission.

\section{Brightness Oscillations at $1600 \AA$}

Cook and Ewing (1991) also examined the brightness variations occuring in HRTS $\mathrm{V} 1600 \AA$ data in a series of 56 images over $330 \mathrm{~s}$ of the sounding rocket flight. We found variations in small 10 arcsec patches in cell centers, which were immediately reminiscent of the five minute oscillations in the photosphere, and the oscillations observed by Noyes and Hall (1972) in the infrared line of $\mathrm{CO}$ at $4.67 \mu \mathrm{m}$ which is formed in the $T_{\min }$. I show in Figure 4 a sample result obtained by fitting the brightness temperature variations with time in individual 5 or $10 \operatorname{arcsec}$ boxes filling a $350 \times 350 \operatorname{arcsec}^{2}$ field from our time series of $1600 \AA$ images. We used sine functions with individual average temperatures, amplitudes, periods, and phases for each box.

With only $330 \mathrm{~s}$ temporal coverage from our sounding rocket observations, a Fourier analysis ( $\omega-\kappa$ diagram) was inappropriate. Instead we looked at the histogram distributions of the periods, amplitudes, etc., found for the individual 5 or 10 arcsec boxes into which we divided our field. Figures 5 and 6 show the distribution of the periods and the amplitudes found from our sine fits to the variation 


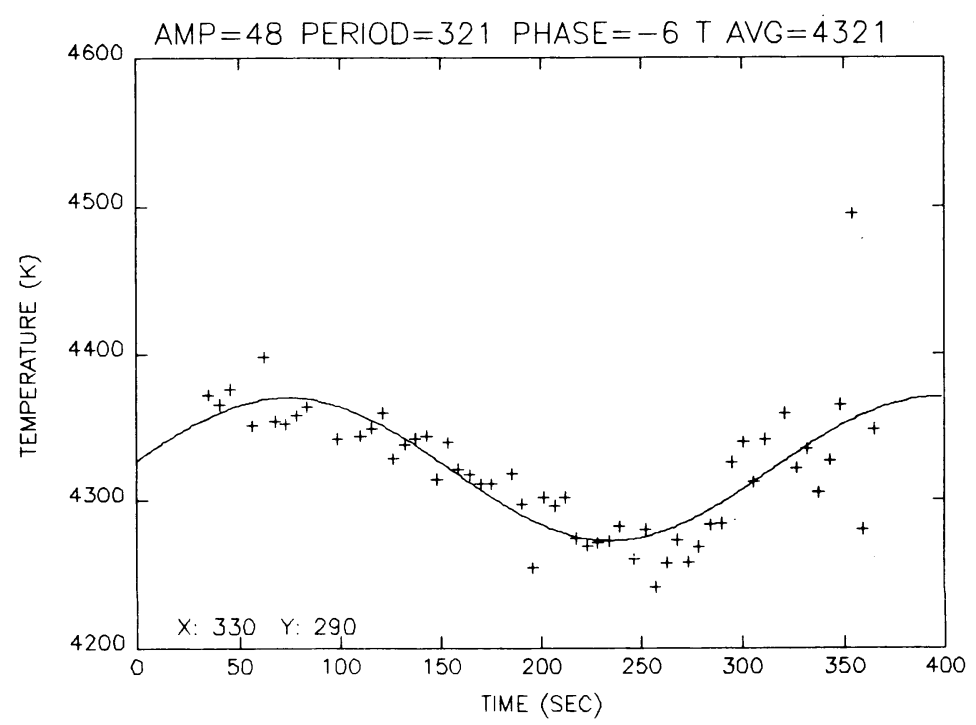

Fig. 4. Sine wave fit to brightness temperature variation for an individual 10 arcsec square box. The scatter to the fit illustrates the noise level of the data.

of brightness temperature within individual boxes. The periods show a broad peak running from 150 to $400 \mathrm{~s}$, centered at $250 \mathrm{~s}$. A range of brightness temperature amplitudes was found, with the average of the $5 \operatorname{arcsec}$ box distribution at $50 \mathrm{~K}$.

These results from our $1600 \AA$ observations can be compared with $T_{\min }$ observations in the infrared, and also with Ca II $\mathrm{K}$ line observations. Observations of $\mathrm{CO}$ infrared absorption lines by Noyes and Hall (1972) and Ayres and Brault (1990) give periods near 250-300 s for oscillations, but significantly greater values of 100$150 \mathrm{~K}$ for the amplitudes. Observations by Lindsey and Roellig (1987) at $350 \mu \mathrm{m}$ also give periods near $300 \mathrm{~s}$; their amplitudes are not really directly comparable because of their large field of view (beam size). In contrast, observations using the Ca II K line (for example Dame, Gouttebroze, and Malherbe 1984) typically give periods in the three minute range in cell centers, which is usually interpreted as a chromospheric period. It is unclear how to directly reconcile the $1600 \AA$, infrared, and $\mathrm{Ca}$ II results into a single picture. The resolution of these differences may come from a model for the temperature minimum and low chromosphere which is highly structured and possibly dynamic.

We estimated the average energy flux $(1 / 2) \rho v^{2} c_{s}$, where the sound speed $c_{s}=$ $\left(\gamma k T / \mu m_{H}\right)^{1 / 2}$, for a simple propagating, adiabatic, undamped wave (however, it is not clear that these waves are actually propagating!). Other regions of the atmosphere depart from adiabaticity, but in the $T_{\min }$ the radiative relaxation time for radiative damping of compressional waves is usually estimated to be long compared with the compression time of an acoustic wave; in addition $\gamma$ should be close to the monatomic ideal value of $5 / 3$ because of the low degree of ionization. In this case 


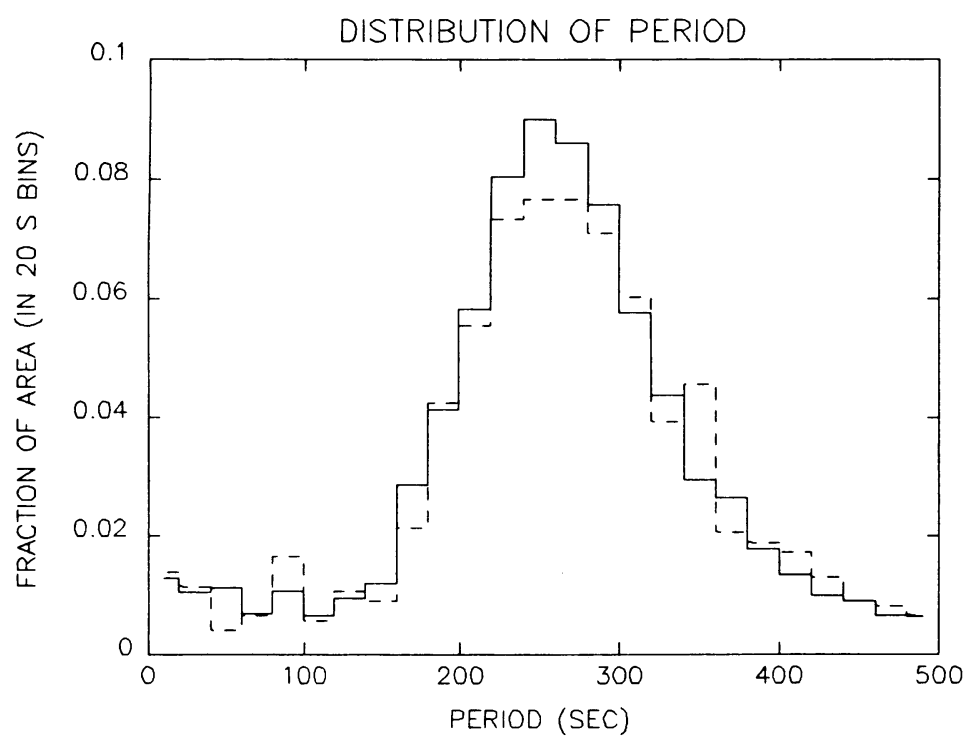

Fig. 5. Histogram distribution of period for 5 arcsec boxes (solid line) and for 10 arcsec boxes (dashed line).

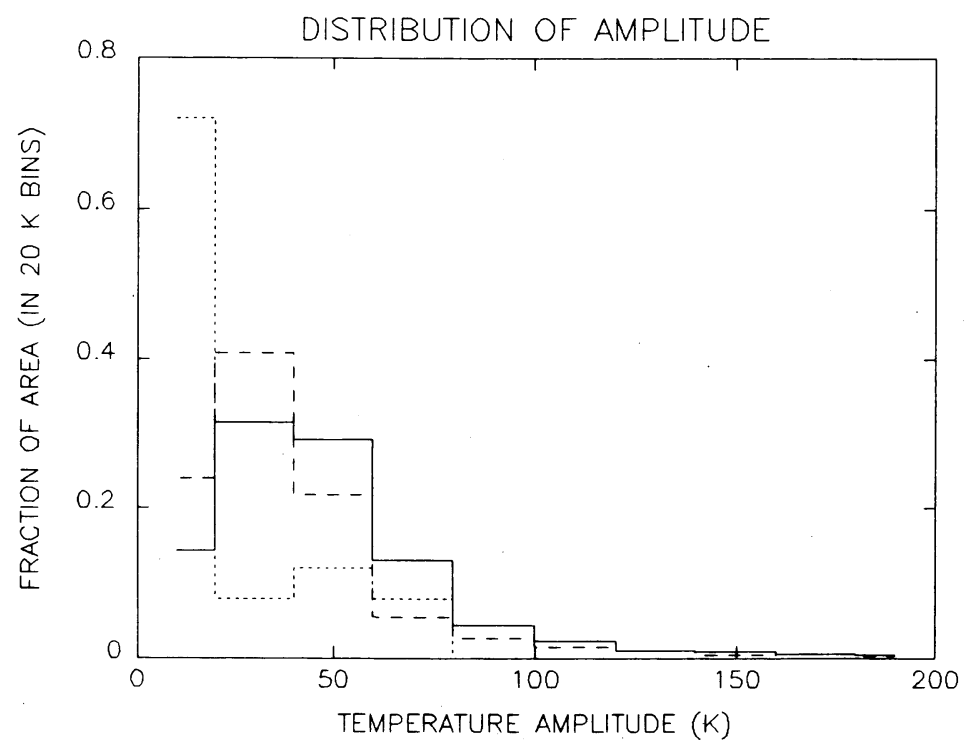

Fig. 6. Histogram distribution of amplitude for 5 arcsec boxes (solid line), 10 arcsec boxes (dashed line), and 70 arcsec boxes (fine dashed line). 
$\left(v / c_{s}\right)=(\gamma-1)^{-1}(\Delta T / T)$, and using $\Delta T=50 \mathrm{~K}$ gives an acoustic flux of $1.8 \times 10^{5}$ ergs $\mathrm{cm}^{-2} \mathrm{~s}^{-1}$. This flux is small compared to an average global requirement of $8 \times 10^{6}$ ergs $\mathrm{cm}^{-2} \mathrm{~s}^{-1}$ to heat the $T_{\min }$ level (Anderson and Athay 1990), and so the 250-s oscillations are energetically unimportant in the local heating budget.

To summarize these results, we suggested a picture for the quiet Sun in which a general nonmagnetic background heating and $250 \mathrm{~s}$ oscillations occur globally, while additional heating (perhaps from partial dissipation of the flux in Alfvén waves produced from photospheric granular buffeting of individual flux tubes) produces the network bright points. These bright points may be individual elemental $\sim 1800 \mathrm{G}$ (at the photosphere) flux tubes which expand to arcsec diameter with decreasing gas pressure by the temperature minimum level. The average additional energy flux required to maintain the network bright points, representing only around $2 \%$ of the total surface area, is directly comparable to the flux required to heat the normal global average background at the temperature minimum level.

The relationship of brightness at $1600 \AA$ and magnetic flux in the photosphere must eventually flatten out above $50 \mathrm{G}$, as finally active region field strengths are reached. Already the brightest few elements in quiet areas are as bright as any features seen at $1600 \AA$ except for flares, and active regions simply contain a higher fractional area of these brighter elements. When field strengths characteristic of sunspots are reached, a presumably completely different physical mechanism takes over to actually suppress heating.

\section{Acknowledgements}

This work was supported by NASA under DPR W-14,541 and by the Office of Naval Research.

\section{References}

Anderson, L.S., and Athay, R.G.: 1990, Astrophys. J. 346, 1010.

Ayres, T.R., and Brault, J.W.: 1990, Astrophys. J. 363, 705.

Cook, J.W.: 1991, in P. Ulmschneider, E.R. Priest, and R. Rosner (eds.), Mechanisms of Chromospheric and Coronal Heating, Springer, Berlin, p. 83.

Cook, J.W., Brueckner, G.E., and Bartoe, J.-D.F.: 1983, Astrophys. J. (Letters) 270 , L89.

Cook, J.W., and Ewing, J.A.: 1990, Astrophys. J. 355, 719.

Cook, J.W., and Ewing, J.A.: 1991, Astrophys. J. 371, 804.

Cook, J.W., Ewing, J.A., and Sutton, C.S.: 1988, Pub. A.S.P. 100, 402.

Dame, L., Gouttebroze, P., and Malherbe, J.-M.: 1984, Astron. Astrophys. 130, 331.

Lindsey, C.: 1993, these proceedings and private communication.

Lindsey, C., and Roellig, T.: 1987, Astrophys. J. 313, 877.

Noyes, R.W., and Hall, D.N.: 1972, Astrophys. J. (Letters) 176, L89.

Sheeley, Jr., N.R., Bohlin, J.D., Brueckner, G.E., Purcell, J.D., Scherrer, V., and Tousey, R.: 1975, Solar Phys. 40, 103.

Title, A., Tarbell, T., Topka, K., Cauffman, D., Balke, C., and Scharmer, G.: 1990, in C.T. Russell, E.R. Priest, and L.C. Lee (eds.), Physics of Magnetic Flux Ropes, American Geophysical Union, Washington, p. 171.

Title, A.M., Tarbell, T.D., Topka, K.P., Ferguson, S.H., Shine, R.A., and the SOUP Team: 1989, Astrophys. J. 336, 475.

Vernazza, J.E., Avrett, E.H., and Loeser, R.: 1981, Astrophys. J. Suppl. 45, 635. 